\title{
HISTOLOGIA MAMÁRIA APÓS USO DE ESTERÓIDES SEXUAIS - ESTUDO EM RATAS
}

\author{
José Tadeu Vicelli, Maria Salete Costa Gurgel*, Marcelo Alvarenga
}

Trabalho realizado no Centro de Atenção Integral à Saúde da Mulher (CAISM) da Universidade Estadual de Campinas (Unicamp) e defendido como Tese de Doutorado pelo Departamento de Tocoginecologia da Faculdade de Ciências Médicas da Unicamp

\author{
*Correspondência: \\ Departamento de \\ Tocoginecologia - \\ FCM/Unicamp \\ Rua Alexander Flemming, \\ 101, Cidade Universitária \\ "Zeferino Vaz", \\ Barão Geraldo \\ Cep: 13083-970, \\ Campinas, SP, \\ Fone/fax: (19) 3788-9305 \\ salete@caism.unicamp.br
}

\section{RESUMO}

OBjetivo. Avaliar as alterações histológicas em mamas de ratas submetidas à terapêutica com estrogênio, progestogênio e tibolona.

Métodos. Estudo experimental com 40 ratas, sendo 20 sem prole (grupo A) e 20 com prole (grupo B). Todas as ratas foram castradas e, após quatro semanas, alocadas aleatoriamente em subgrupos: $A 1, A 2, A 3, A 4, A 5$ e BI, B2, B3, B4, B5. Os esteróides foram administrados da seguinte forma: $A$ l e BI - benzoato de estradiol; $A 2$ e $B 2$ - acetato de medroxiprogesterona; $A 3$ e B3 - benzoato de estradiol e acetato de medroxiprogesterona; A4 e B4 - tibolona; A5 e B5 placebo. Após dez semanas de tratamento, os animais foram sacrificados e suas glândulas mamárias submetidas à análise histológica. Os parâmetros avaliados foram: proliferação epitelial, atividade secretora e atipias epiteliais nas unidades de ductos ou alvéolos terminais. A associação entre os achados histológicos e os esquemas terapêuticos foi avaliada por meio do odds ratio e intervalo de confiança de $95 \%$.

Resultados. Alterações histológicas foram observadas em 29 ratas: hiperplasia moderada (52,5\%), hiperplasia alvéolo-nodular (42,5\%), atipia sem proliferação (35\%), hiperplasia leve (32,5\%), atividade secretora (20\%) e hiperplasia severa (5\%). Em ratas sem prole observou-se 1,3 mais chance, em relação ao grupo controle, de apresentar hiperplasia alvéolo-nodular no grupo que recebeu estrogênio, hiperplasia moderada no grupo tratado com progestogênio, e hiperplasia alvéolo-nodular e atipia sem proliferaçãa epitelial com a associação entre estrogênio e progestogênio.

Conclusäo. Hiperplasia moderada e atipia epitelial associaram-se à terapia combinada de estrogênio e progestogênio, e o antecedente de prole reduziu a ocorrência destas alterações e de hiperplasia alvéolo-nodular.

UnITERMos: Mama. Proliferação epitelial. Esteróides sexuais. Atipia epitelial.

\section{INTRODUÇÃO}

Nas últimas décadas, estudos demonstraram os benefícios da terapia hormonal $(\mathrm{TH})$ no climatério, representados pelo alívio de sintomas, prevenção e tratamento da atrofia urogenital e da osteoporose ${ }^{1,2,3}$. A despeito dos benefícios da $\mathrm{TH}$, o seu uso pode apresentar alguns efeitos indesejáveis, como o aumento do risco de câncer de mama ${ }^{4,5,6}$. Existem diversos esquemas de TH, porém os mais utilizados são o uso exclusivo de estrogênio ou a associação deste com progestogênio.

Além destes esteróides, a tibolona - um esteróide sintético vem sendo utilizada para o tratamento dos sintomas climatéricos, possuindo ações estrogênica, progestogênica e androgênica?. Estudos experimentais demonstraram que, in vitro, a tibolona reduz a proliferação de células mamárias e a produção de estradio ${ }^{8}$, enquanto in vivo reduz o volume tumoral de carcinomas mamários?. Contudo, estudos de coorte demonstraram aumento do risco relativo para desenvolvimento de câncer de mama em usuárias de tibolona ${ }^{6,10}$. Dessa forma, permanece incerto o papel da tibolona na indução de alterações proliferativas e no risco de câncer de mama.

Hofseth et al. (1999)", estudando biópsias de mamas de mulheres na pós-menopausa, observaram que a proliferação do epitélio mamário foi maior em usuárias de $\mathrm{TH}$, principalmente em usuárias de estrogênio e progestogênio, quando comparadas a um grupo de não usuárias de TH. Nesse mesmo estudo, verificou-se que a maioria das alterações localizava-se na unidade ducto-lobular terminal, onde se desenvolve a maior parte dos cânceres de mama. Estudos experimentais também verificaram alterações proliferativas nas mamas de ratas ooforectomizadas submetidas a $\mathrm{TH}^{\prime 2}$.

As glândulas mamárias humanas e da rata exibem alterações proliferativas comparáveis quando submetidas a $\mathrm{TH}$, suportando a validade do estudo dessas alterações no modelo murino na pós-menopausa ${ }^{12}$. O uso do modelo animal permite a avaliação do efeito direto da $\mathrm{TH}$ sobre o tecido mamário, eliminando-se os efeitos de potenciais variáveis confundidoras que podem estar presentes em ensaios clínicos como idade, tempo de menopausa e tempo de uso de $\mathrm{TH}^{13}$.

Considerando esses aspectos, realizou-se o presente estudo com o objetivo de avaliar a associação de alterações histológicas epiteliais nas mamas de ratas castradas após tratamento exclusivo com estrogênio, progestogênio e tibolona e com a associação de estrogênio-progestogênio segundo o esquema terapêutico, e se o antecedente de prole interfere nesta associação. 
VICELLI JT ET AL.

\section{Métodos}

Trata-se de estudo experimental, no qual foram utilizadas 40 ratas (Rattus norvegicus) da linhagem Wistar com 250 dias de vida, sendo que 20 já procriaram e amamentaram suas proles (grupo B), enquanto as outras 20 não tiveram prenhez (grupo A). As ratas foram castradas sob anestesia geral com injeções intraperitoniais de cetamida (Vetaset ${ }^{\mathrm{R}}$, Fort Dodge Laboratories) e xilazina (Rompum ${ }^{R}$, Bayer) ambas na dose de $50 \mathrm{mg} / \mathrm{kg}^{14,15}$, ficando em observação por três semanas a fim de esgotar a ação dos esteróides ovarianos endógenos e entrarem em fase de hipoestrogenismo, permitindo que o tecido mamário fique em repouso ${ }^{16}$. O hipoestrogenismo foi confirmado antes do início da terapêutica pela realização de esfregaços vaginais ${ }^{17}$.

Os tratamentos hormonais foram realizados por dez semanas, sendo que as dosagens foram calculadas segundo tabela de cálculo halométrico, que converte a dose utilizada em humano para a correspondente na espécie animal utilizada no estudo: I-benzoato de estradiol $\left(E_{2}\right), 37,6 \mathrm{mg} / \mathrm{rata} / 7$ dias diluído em óleo de amendoim estéril, que é o veículo original do produto, de tal forma que a dosagem está contida em $0,1 \mathrm{ml}$ da solução; 2-acetato de medroxiprogesterona na forma de depósito na dosagem de I I, 28mg/rata/23 dias (AMP); diluído em solução fisiológica, de tal forma que a dosagem está contida em 0, I ml da solução final; 3-benzoato de estradiol $\left(E_{2}\right)$ $37,6 \mathrm{mg} / \mathrm{rata} / 7$ dias + acetato de medroxiprogesterona na forma de depósito na dosagem de I I, 28 mg/rata/23 dias (AMP); 4-tibolona, $200 \mathrm{mg} / \mathrm{rata} / \mathrm{dia}$. Os estrogênios e progestogênios foram administrados por via subcutânea na região dorsal dos animais, uma vez ao dia. A tibolona foi administrada por via oral, uma vez ao dia, diluída em água para ser administrada por gavagem.

Cada animal foi alocado, aleatoriamente, em dez subgrupos de quatro ratas ( $A 1, A 2, A 3, A 4, A 5$ e $B 1, B 2, B 3, B 4$, e B5) e assim designados segundo o tipo de tratamento: $A \mid$ e $B \mid$ - benzoato de estradiol e placebo para o AMP (solução fisiológica); A2 e B2 - acetato de medroxiprogesterona e placebo para $E_{2}$ (óleo de amendoim); $A 3$ e B3 - benzoato de estradiol e acetato de medroxiprogesterona; A4 e B4 - tibolona e placebo (óleo de amendoim e solução fisiológica); A5 e B5 - placebo (solução fisiológica e óleo de amendoim).

Os animais permaneceram no biotério do Laboratório de Pesquisas Bioquímicas do Centro de Atenção Integral à Saúde da Mulher (CAISM) da Universidade Estadual de Campinas (Unicamp) em caixas com iluminação de 12 horas/dia e temperatura constante, além de água e alimentação ad libitium. Os quatro animais de cada subgrupo foram mantidos em uma única caixa com cuidados diários e limpeza. Ao final de dez semanas, todas as ratas foram sacrificadas em câmara de $\mathrm{CO}_{2}{ }^{18}$. Em seguida foram dissecadas e removidas as segundas glândulas mamárias torácicas, juntamente com a pele que as recobre. Todo o material obtido foi imediatamente fixado em formalina a 10\%, tamponado $(\mathrm{pH} 7,4)$ e enviado ao Laboratório de Patologia Experimental do CAISM/ Unicamp. Após 24 horas de fixação, o tecido mamário foi recortado, submetido ao processamento histológico, incluído em blocos de parafina e recortado em micrótomo de $4 \mathrm{~m}$ de espessura, colocado em várias lâminas e corado pela técnica de hematoxilina-eosina (HE).
Foram avaliados todos os blocos correspondentes à totalidade das mamas de cada rata pelo mesmo patologista, sem que este conhecesse a terapêutica a que cada rata havia sido submetida; utilizou-se um microscópio Nikon E 200 com objetiva de 40 vezes. Foram analisados a proliferação epitelial e atipias celulares nas unidades de ductos terminais e os brotos ou alvéolos terminais, sendo que o exame foi realizado baseando-se em um sistema de graduação histopatológico para anomalias epiteliais induzidas por 7, 12-dimetilbenzantraceno (DMBA) em tecido mamário de fêmeas de ratos. O sistema de graduação histopatológica referente à hiperplasia epitelial e células epiteliais com atividade secretora seguiu critérios padronizados e estabelecidos previamente ${ }^{19}$, e foi classificado conforme a seguinte descriçãa: normal; atipia sem proliferação epitelial; HEB I - hiperplasia epitelial da unidade ductal terminal lobular leve; HEB 2 - hiperplasia epitelial da unidade ductal terminal lobular moderada; HEB 3 hiperplasia epitelial da unidade ductal terminal lobular severa; HAN hiperplasia alvéolo-nodular; atividade secretora em células epiteliais. Para a análise dos dados foram feitas tabelas de contingência e calculado o odds ratio com respectivo intervalo de confiança de $95 \%$, sendo utilizado o programa de computador SAS, 2001.

Este estudo seguiu as normas da Comissão de Ética na Experimentação Animal (CEEA) do Instituto de Biologia (IB) da Unicamp, sendo aprovado pelo protocolo de $n^{0} 65 \mathrm{I}-\mathrm{I}$.

\section{Resultados}

Ao final de dez semanas de terapia hormonal ou placebo, 29 ratas apresentaram alterações epiteliais focais em suas glândulas mamárias, sendo: hiperplasia epitelial da unidade ductal terminal lobular leve ou HEB I em I3 casos (32,5\%), hiperplasia epitelial da unidade ductal terminal lobular moderada ou HEB 2 em 2 I casos (52,5\%), hiperplasia epitelial da unidade ductal terminal lobular severa ou HEB 3 em dois casos (5\%), hiperplasia alvéolo-nodular ou HAN em 17 casos (42,5\%), atipia sem proliferação epitelial em 14 casos (35\%) e atividade secretora (AS) em oito casos (20\%). Onze ratas (27,5\%) apresentavam mamas histologicamente normais ao final do estudo (Figura I).

Analisando as alterações histológicas segundo o antecedente de prole e de acordo com o esquema terapêutico, observou-se que, no grupo sem prole, no subgrupo tratado com E2, todas as ratas desenvolveram HEB 2 ou 3, HAN e um caso apresentou atipia epitelial sem proliferação. No subgrupo tratado com AMP isoladamente, todas as ratas apresentaram HEB 2, três delas atipia sem proliferação epitelial, dois casos de HAN e dois casos de atividade secretora. No subgrupo tratado com a associação de estrogênio e progestogênios, todas as ratas desenvolveram HEB 2 ou 3, HAN e atipia sem proliferação epitelial. No subgrupo tratado com tibolona, observaram-se duas ratas sem alterações mamárias e as outras duas apresentaram HEB 2, atipia sem proliferação e atividade secretora. No grupo placebo, três ratas apresentaram apenas foco único de HEB I (Tabela I).

Analisando as alterações histológicas observadas no grupo com prole (grupo B), notamos que, com o tratamento exclusivo com estrogênio, sete ratas desenvolveram HEB | ou 2 e quatro delas apresentaram atividade secretora. Nenhuma atipia celular foi observada. As ratas tratadas com AMP exclusivo apresentaram padrão 
Tabela I - Distribuição das alterações histológicas em mamas de ratas de acordo com a terapêutica com esteróides sexuais e segundo 0 antecedente de prole $(n=40)$

\begin{tabular}{|c|c|c|c|c|c|c|c|c|c|c|c|c|}
\hline \multirow[t]{2}{*}{ Alterações Histológicas } & \multicolumn{2}{|c|}{$\frac{\mathbf{E}_{2}}{\text { Prole }}$} & \multicolumn{2}{|c|}{$\frac{\text { AMP }}{\text { Prole }}$} & \multicolumn{2}{|c|}{$\frac{E_{2}+\text { AMP }}{\text { Prole }}$} & \multicolumn{2}{|c|}{$\frac{\mathrm{T}}{\text { Prole }}$} & \multicolumn{2}{|c|}{$\frac{\text { Placebo }}{\text { Prole }}$} & \multicolumn{2}{|c|}{$\frac{\text { Total }}{\text { Prole }}$} \\
\hline & $\begin{array}{l}\text { Sem } \\
(4)\end{array}$ & $\begin{array}{l}\text { Com } \\
(4)\end{array}$ & $\begin{array}{l}\text { Sem } \\
(4)\end{array}$ & $\begin{array}{c}\text { Com } \\
(4)\end{array}$ & $\begin{array}{l}\text { Sem } \\
(4)\end{array}$ & $\begin{array}{c}\text { Com } \\
\text { (4) }\end{array}$ & $\begin{array}{l}\text { Sem } \\
(4)\end{array}$ & $\begin{array}{c}\text { Com } \\
(4)\end{array}$ & $\begin{array}{c}\text { Sem } \\
(4)\end{array}$ & $\begin{array}{c}\text { Com } \\
\text { (4) }\end{array}$ & $\begin{array}{l}\text { Sem } \\
(20)\end{array}$ & $\begin{array}{l}\text { Com } \\
(20)\end{array}$ \\
\hline Normal & 0 & 0 & 0 & 0 & 0 & 0 & 2 & I & I & 4 & 3 & 5 \\
\hline HEB I & 0 & 4 & 0 & 3 & 0 & 0 & 0 & 3 & 3 & 0 & 3 & 10 \\
\hline HEB 2 & 3 & 3 & 4 & 2 & 3 & 4 & 2 & 0 & 0 & 0 & 12 & 9 \\
\hline HEB 3 & । & 0 & 0 & 0 & 1 & 0 & 0 & 0 & 0 & 0 & 2 & 0 \\
\hline Atipia & । & 0 & 3 & l & 4 & 4 & । & 0 & 0 & 0 & 9 & 5 \\
\hline HAN & 4 & 0 & 2 & 3 & 4 & 4 & 0 & 0 & 0 & 0 & 10 & 7 \\
\hline AS & 0 & 4 & 2 & I & 0 & 0 & । & 0 & 0 & 0 & 3 & 5 \\
\hline
\end{tabular}

HEB - hiperplasia epitelial da unidade ductal terminal lobular HAN - hiperplasia alvéolo-nodular

\section{Figura I - Alterações histológicas observadas em mamas de ratas castradas e submetidas à terapêutica com esteróides sexuais e tibolona. A- HEB I; B- HEB 2; C- HEB 3; D- atipia sem proliferação epitelial; E- HAN; e F- atividade secretora}
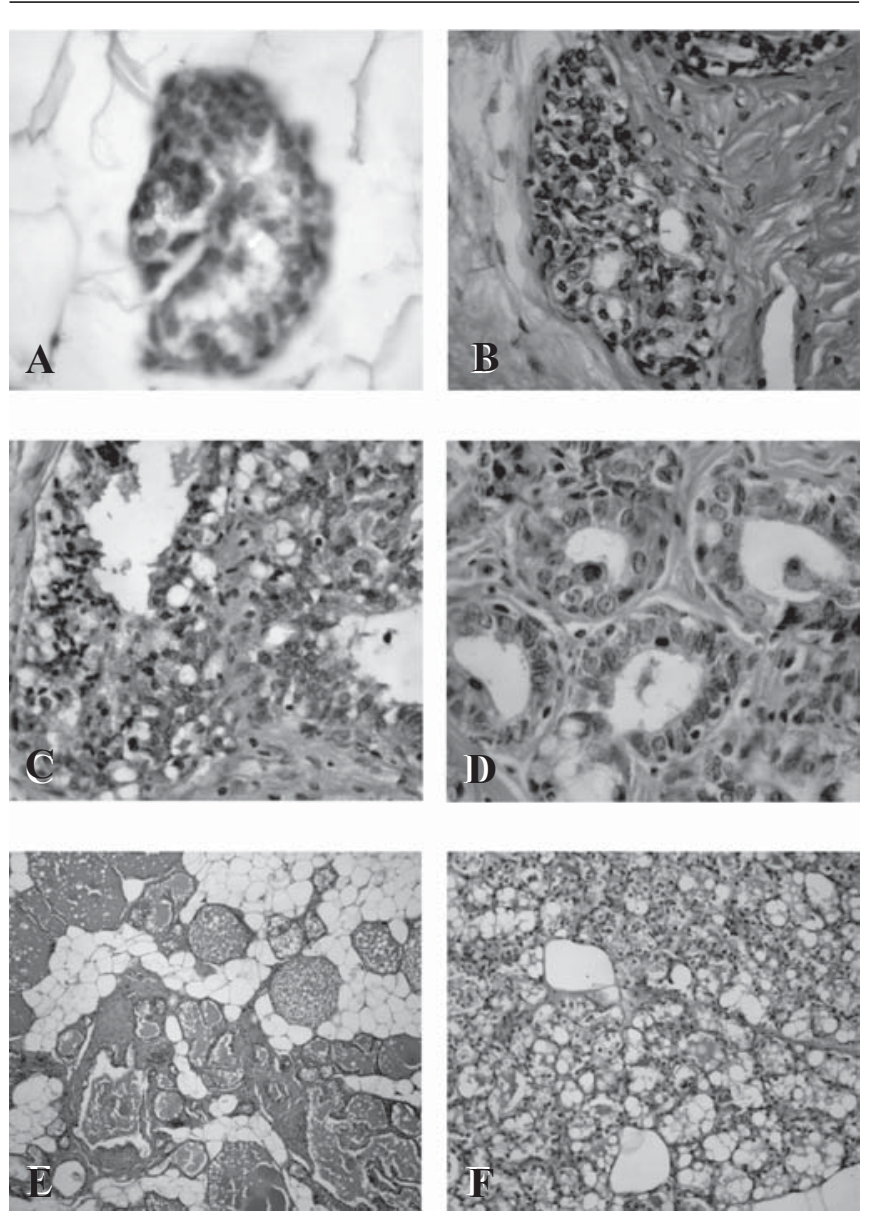

semelhante em relação às HEB, porém houve três casos de HAN e uma rata desenvolveu atipia sem proliferação epitelial. Já no subgrupo tratado com associação de estrogênio com progestogênios, observaram-se índices de 100\% para a HEB 2, HAN e atipia sem proliferação. As ratas tratadas com tibolona desenvolveram apenas HEB I em três casos e 100\% das ratas no subgrupo placebo tinham mamas histologicamente normais (Tabela I).

Ao se avaliar as chances de desenvolvimento das alterações histológicas segundo o esquema terapêutico, observou-se que dentre as ratas sem prole (grupo A), aquelas expostas isoladamente ao E2 tiveram, pelo menos, I,3 mais chance de desenvolver HAN em comparação ao grupo controle. Aquelas submetidas à terapêutica com AMP isolado tiveram aumento da chance de mesma magnitude para desenvolvimento de HEB 2. Já as ratas tratadas com E2 + AMP tiveram aumento significativo, e de mesma magnitude, para o desenvolvimento de atipias sem proliferação epitelial e HAN. O subgrupo tratado com tibolona não apresentou aumento da chance de desenvolver qualquer alteração histológica mamária em relação ao grupo controle (Tabela 2). A mesma análise feita no grupo de ratas com prole (grupo B) demonstrou haver associação entre a terapêutica de estrogênio e progestogênios combinados com a presença de atividade secretora nas células epiteliais (Tabela 3).

\section{Discussão}

No presente estudo houve associação do uso de esteróides sexuais com proliferação e atipias epiteliais e, ainda, atividade secretora celular, alterações essas observadas em animais que receberam terapia isolada com estrogênios ou progestogênios, embora o maior efeito tenha sido observado com a combinação de ambos. Estas alterações epiteliais, apesar de se basearem em anomalias induzidas por DMBA em tecido mamário de fêmeas de ratos ${ }^{19}$, têm sido utilizadas na maioria dos estudos in vivo que utilizam o modelo murino ${ }^{12,20}$.

É possível fazer uma analogia entre as alterações descritas nas mamas de ratas com as observadas na mama humana. A HEB, graduada 
Tabela 2 - Alterações histológicas em mamas de ratas sem prole submetidas à terapêutica com esteróides sexuais ( $n=20)$ - OR (IC 95\%)

\begin{tabular}{|c|c|c|c|c|c|}
\hline \multirow[b]{2}{*}{ Alteraçōes histológicas } & \multicolumn{5}{|c|}{ Tratamentos } \\
\hline & $\begin{array}{c}E_{2} \\
(n=4)\end{array}$ & $\begin{array}{l}\text { AMP } \\
(n=4)\end{array}$ & $\begin{array}{c}E_{2}+A M P \\
(n=4)\end{array}$ & $\underset{(n=4)}{\mathbf{T}}$ & $\begin{array}{r}\text { Placebo } \\
(n=4)\end{array}$ \\
\hline HEB I & - & - & - & - & 1,0 \\
\hline HEB 2 & $21,0(0,6-690,0)$ & $81,0(1,3-5046,7)$ & $21,0(0,6-690,0)$ & $9,0(0,3-271,7)$ & 1,0 \\
\hline HEB 3 & $3,9(0,1-\mid 26,7)$ & - & $3,9(0,1-126,7)$ & - & 1,0 \\
\hline Atipia & $3,9(0,1-126,7)$ & $21,0(0,6-690,0)$ & $81,0(1,3-5046,7)$ & $3,9(0,1-126,7)$ & 1,0 \\
\hline HAN & $81,0(1,3-5046,7)$ & $9,0(0,3-271,7)$ & $81,0(1,3-5046,7)$ & - & 1,0 \\
\hline AS & - & $9,0(0,3-271,7)$ & - & $3,9(0,1-126,7)$ & 1,0 \\
\hline
\end{tabular}

HEB - hiperplasia epitelial da unidade ductal terminal lobular

HAN - hiperplasia alvéolo-nodular

AS - atividade secretora

Tabela 3 - Alterações histológicas em mamas de ratas com prole submetidas à terapêutica com esteróides sexuais $(n=20)$ - OR (IC 95\%)

\begin{tabular}{|c|c|c|c|c|c|}
\hline \multirow[b]{2}{*}{ Alterações histológicas } & \multicolumn{5}{|c|}{ Tratamentos } \\
\hline & $\begin{array}{c}E_{2} \\
(n=4)\end{array}$ & $\begin{array}{l}\text { AMP } \\
(n=4)\end{array}$ & $\begin{array}{c}\mathrm{E}_{2}+\mathrm{AMP} \\
(\mathrm{n}=4)\end{array}$ & $\underset{(n=4)}{T}$ & $\begin{array}{l}\text { Placebo } \\
(n=4)\end{array}$ \\
\hline HEB $\mid$ & $3,9(0,1-126,7)$ & $21,0(0,6-690,0)$ & - & $21,0(0,6-690,0)$ & 1,0 \\
\hline HEB 2 & $21,0(0,6-690,0)$ & $9,0(0,3-271,0)$ & $3,9(0,1-126,7)$ & - & 1,0 \\
\hline HEB 3 & - & - & - & - & 1,0 \\
\hline Atipia & - & $3,9(0,1-32,3)$ & $3,9(0,1-126,7)$ & - & 1,0 \\
\hline HAN & - & $21,0(0,6-690,0)$ & $81,0(1,3-5046,7)$ & - & 1,0 \\
\hline AS & $81,0(1,3-5046,7)$ & $3,9(0,1-32,3)$ & - & - & 1,0 \\
\hline
\end{tabular}

HEB - hiperplasia epitelial da unidade ductal terminal lobular

HAN - hiperplasia alvéolo-nodular

AS - atividade secretora

em leve, moderada e grave ou severa, corresponderia na mulher: HEB I a hiperplasia ductal tíica (HDT) leve, alteração considerada sem risco para câncer invasor; a HEB 2 a HDT moderada, alteração considerada de risco leve (duas vezes) para o câncer invasor; e a HEB 3 a hiperplasia ductal atíica (HDA), lesão associada a risco cinco vezes aumentado para o câncer invasor da mama²!

Observou-se, também, a presença de atipias celulares sem proliferação epitelial ainda não descritas em ratas, muito semelhantes às alterações designadas de atipia plana na mama humana. Esta entidade é caracterizada por células epiteliais nativas constituídas em camadas simples ou por três a cinco camadas e com atipias leves. Segundo a classificação da Organização Mundial da Saúde, seria uma lesão intermediária entre a HDT e HDA ${ }^{21}$.

A atipia epitelial plana tem sido denominada por outros patologistas como alteração ou hiperplasia de células colunares com atipias ${ }^{22-23}$ ou ainda CAPSS - columnar alteration with prominent apical snouts and secretions ${ }^{24}$ (alteração colunar epitelial com secreção do tipo decapitação $0^{25}$ ). Atualmente, o nível de risco para o desenvolvimento de carcinoma invasivo associado com estas lesões é desconhecido. No entanto, já foi verificado que o risco relativo das lesões colunares em geral foi 2,08 vezes maior que os controle e, para as lesões colunares com atipia, o risco foi de 2,3226.

A hiperplasia alvéolo-nodular (HAN), lesão epitelial caracterizada por área nodular bem delimitada, constituída de grupamentos de dúctulos e alvéolos terminais, cujas células apresentam citoplasma vacuolizado semelhante a células apócrinas, apesar da denominação "hiperplasia", acredita-se que reflita atividade secretora. É alteração descrita apenas em ratas e sem correspondência na mama humana ${ }^{19}$. No entanto, estudos mais recentes em modelo murino evidenciaram associação dessa entidade com o carcinoma mamário induzido por DMBA, podendo ser considerada como potencial lesão precursora de neoplasias ${ }^{27}$.

Estudos prévios sobre a ação dos esteróides sexuais em mamas de ratas utilizaram doses variadas e muitas vezes elevadas ${ }^{28}$. Como se sabe, o estrogênio pode ser mitogênico em doses elevadas ${ }^{29}$. Essa pesquisa objetivou descrever as alterações histológicas nas mamas submetidas a doses análogas às usadas por mulheres climatéricas, por meio de cálculo halométrico, equacionando a dose de acordo com 
o peso e o metabolismo animal ${ }^{30-31}$. Além disso, as ratas foram selecionadas de tal forma que o número de ciclos estrais correspondessem aos da mulher no menacme.

Neste estudo houve associação dos estrogênios com a hiperplasia das células epiteliais mamárias. Isto ficou demonstrado no grupo de ratas que usou estrogênio apresentando muito mais HEB que o grupo controle. Não se observou associação dos estrogênios isoladamente com atipias celulares em qualquer dos grupos de animais, com ou sem prole, embora na literatura existam estudos que evidenciam ação genotóxica do 17 B estradiol sobre as células mamárias ${ }^{32,33}$.

Em estudos experimentais com modelos animais observou-se que a progesterona não é protetora da carcinogênese mamária, evidenciando a ausência de bloqueio da atividade proliferativa estrogênica por ação do progestogênio, aliada ao estímulo proliferativo epitelia|l,34,36. Observou-se, ainda, maior atividade mitótica na fase lútea29,37,38.

Os resultados deste estudo mostram que o progestogênio, quando utilizado isoladamente, também causou aumento da atividade proliferativa celular mamária equivalente ao grupo que usou estrogênio. Isso leva a inferir que o progestogênio potencializaria o efeito estrogênico e que não haveria necessidade da ação estrogênica prévia para sua ação. Estudos prévios observaram que a administração de progestogênio induz maior proliferação de células acinares e atividade mitótica, à semelhança da ação estrogênica, propondo que o estrogênio atuaria estimulando a proliferação celular e o progestagênio faria o mesmo, além de induzir a diferenciação quando há ação estrogênica 39,40,41,42.

Os esteróides sexuais, mesmo que acarretem a diferenciação celular ao estimularem a atividade proliferativa mamária, podem propiciar alterações carcinogenéticas, celulares. Assim, se os processos de proliferação e diferenciação mamária ocorrerem antes da ação do agente carcinogênico, diminui-se a chance de desenvolvimento do câncer no futuro ${ }^{43}$. Isto explicaria o fato do AMP administrado em ratas na fase puberal diminuir o risco de desenvolvimento tumoral, enquanto a administração após essa fase não propicia o mesmo efeito protetor ${ }^{44}$. $O$ mesmo explicaria a proteção conferida pela primeira gestação a termo em idade precoce. Neste estudo, o fato de ter prole parece ter influenciado de forma protetora a resposta celular aos esteróides sexuais.

A tibolona, neste estudo, não mostrou associação com hiperplasia ou atipia epitelial mamária. Esses resultados encontram respaldo em estudos sobre a ação da tibolona em células mamárias normais obtidas de mastoplastia redutora, de mulheres com idade inferior a 25 anos. Após sete dias sob ação da tibolona, ocorreu lenta diminuição na taxa de proliferação das células epiteliais ${ }^{45}$.

Muito embora este estudo tenha objetivado avaliar as alterações histológicas em mamas submetidas à terapêutica hormonal, podemos inferir que a proliferação celular, assim como as atipias encontradas com a associação de estrogênios e progestogênios e com estrogênios isolados, possam estar relacionadas com risco aumentado para câncer de mama.

A maior contribuição deste estudo talvez seja a de subsidiar outras informações relativas à ação destas substâncias sobre o tecido mamário normal e, com isso, nortear a escolha terapêutica das mulheres na menopausa. Discutem-se atualmente esquemas com associações progestogênicas trimestrais ou quadrimestrais, visando minimizar a exposição a este grupo de esteróide ${ }^{46}$; baixas dosagens de esteróides sexuais e associações de estrogênio com progestogênios de ação específica para seus receptores, e isso deve ser encarado como um incentivo à pesquisa e à disponibilização de substâncias que poderiam agir como progestogênios no endométrio e não na mama, ou seja, seriam moduladores seletivos de receptores de progesterona, à semelhança dos moduladores seletivos de receptores de estrógeno ${ }^{47}$.

\section{Conclusão}

A hiperplasia epitelial e atipia sem proliferação epitelial associaram-se, mais intensamente, à terapia combinada de estradiol e acetato de medroxiprogesterona, principalmente no grupo de ratas sem antecedente de prole.

\section{Conflito de interesse: não há}

\section{SUMMARY}

\section{BREASt histologic Changes in female RATS treated WitH SEX STEROIDS}

OBJECTIVE. To evaluate the association of histologic changes in the breasts of female rats undergoing therapy with sex steroids.

METHODS. An experimental study was conducted of 40 castrated female non-pubertal rats, 20 had given birth (Group B) and 20 had no offspring (Group A). After four weeks, these rats were randomly allocated to subgroups: $A 1, A 2, A 3, A 4, A 5$ and $B 1, B 2, B 3, B 4, B 5$. Steroids were given to subgroups as follows: $A$ I and $B I$ - estradiol benzoate; $A 2$ and $B 2$ - medroxyprogesterone acetate; $A 3$ and $B 3$ estradiol benzoate and medroxyprogesterone acetate; $A 4$ and $B 4$ tibolone; $A 5$ and $B 5$ - placebo. After 10 weeks of treatment, animals were sacrificed and their mammary glands were analyzed. Histologic parameters evaluated were: epithelial cell proliferation, epithelial cells with secretory activity; and cell atypia in terminal duct units and buds or terminal alveoli. The association between microscopic analysis and diverse therapeutic regimens were analyzed by calculating the odds ratio and its respective $95 \%$ confidence interval.

RESULTS. Histologic changes were observed in 29 rats: moderate hyperplasia (52.5\%), hyperplastic alveolar nodule (42.5\%), epithelial atypia (35\%), mild hyperplasia (32.5\%), secretory activity (20\%) and severe hyperplasia (5\%). In rats with no offspring when compared to the control, 1.3 times more hyperplastic alveolar nodules were found in the group treated with estradiol, the same was true for moderate hyperplasia in the rats that received medroxyprogesterone acetate, hyperplastic alveolar nodules and epithelial atypia in the group treated with estradiol plus medroxyprogesterone acetate. In the rats with offspring 1.3 times more secretory activity was found with estradiol.

CONCLUSION. Epithelial hyperplasia and epithelial atypia with no proliferation are strongly associated to combined therapy with estradiol plus medroxyprogesterone acetate, mainly in the rats without offspring. [Rev Assoc Med Bras 2006; 52(5): 369-74]

KEY wORDS: Breast. Epithelial proliferation. Sex steroids. Epithelial atypia.

\section{REFERÊNCIAS}

I. Maclenan A, Lester S, Moore. Oral oestrogen replacement therapy versus placebo for hot flushes (Cochrane Review). In: The Cochrane Libray, Issue 3, 2002. Oxford: Update Software. 
VICELLI JT ET AL.

2. Torgerson DJ, Bell-Ssyer SE. Hormone replacement therapy and prevention of nonvertebral fractures: a meta-analysis of randomized trials. JAMA 200 I;285:289I-7.

3. Oldenhave A, Jaszmann LJ, Haspeles AA, Everaerd WT. Impact of climateric on well-being: a survey based on 5213 women 39 to 60 years old. Am J Obstet Gynecol 1993; 168:772-80.

4. Collaborative Group On Hormonal Factors In Breast Cancer. Breast cancer and hormone replacement terapy; collaborative reanalysis of data from 5 I epidemiological studies 52705 women with breast cancer and I 084 I I women without breast cancer. Lancet 1997;50: I 047-59.

5. Writing Group for the WHI Investigators. Risk and benefits of estrogen plus progestin en healthy post menopausal women: principal results. From the Women's Health Initiative randomized controlled trial. JAMA 2002;288:321-3.

6. Million Women Study Collaborators Breast cancer and hormonereplacement therapy in the Million Women Study. Lancet 2003;362:41 9-27.

7. Rymer JM. The effects of tibolone. Gynecol Endocrinol 1998; I 2:2 13-20.

8. Gompel A, Chaouat M, Jacob D, Perrot JY, Kloosterboer HJ, Rostene W. In vitro studies of tibolone in breast cells. Fertil Steril 2002;78:35I-9.

9. Kloosterboer HJ, Schoonen W, Deckers G. Effects of progestagens and org od 14 in vitro and in vivo tumor models. J Steroid Biochem 1994:49:31।-8.

10. Stahlberg C, Pedersen AT, Lynge E, Andersen ZJ, Keiding N, Hundrup $Y A$, et al. Increased risk of breast cancer following different regimens of hormone replacement therapy frequently used in Europe. Int J Cancer 2004; 109:721-7.

I I. Hofseth LJ, Raafat AM, Osuch JR, Pathack DR, Slomski CA, Haslam SZ. Hormone replacement therapy with estrogen or estrogen plus medroxiprogesterone acetate is associated with increased epithelial proliferation in the normal postmenopausal breast. J Clin Endocrinol Metab 1999;84:4559-65.

12. Russo J, Russo IH. Role of hormones in cancer e initiation and progression. J Mamary Gland Biol Neoplasia 1998;3:49-6I .

13. Clarkson TB, Appt SE, Wood CE, Cline JM. Lessons to be learned from animal studies on hormones and breast. Maturitas 2004;49:79-89.

14. Yang NN, Bryant HU, Hardikar S, Sato M, Galvin RJS, Glasebrook AL, et al. Estrogen and raloxifene stimulate transforming growth factor b3 gene expression in rat bone: a potential mechanism for estrogen-or roloxifene - mediated bone maintenance. Endocrinology 1996; 137:2075-84.

I5. Bolscher MT, Netelenbos JC, Barto R, Buuren LM, Vijgh WJF. Estrogen regulation of intestinal calcium absorption in the intact and ovariectomized adult rat. J Bone Min Res 1999; | 4: 197-202.

16. Ciocca DR. Parente A, Russo J. Endocrinologic milieu and susceptibility of the rat mamary gland to carcinogenesis. Am J Pathol 1982; 109:47-56.

17. Shorr E. A new technic for staining vaginal smears: III, a single differential stain. Science 1941;94:545-6.

18. Black LJ, Sato M, Rowley ER, Magee DE, Bekele A, Willians DC, et al. Raloxifene (LY I3948 I HCL) prevents bone loss and reduces serum cholesterol without causing uterine hipertrophy en ovariectomized rats. J Clin Invest 1994;93:63-9.

19. Alvarenga M, Cavalcanti TC, Tahin QS. Histopathologic grading system for epitelial abnormalities induced by 7,12-dimethylbenz[a]anthracene (DMBA) in female rat mammary tissue . Breast Dis 1989;2:71 -9.

20. Russo J, Russo $\mathbb{I H}$. Experimentally induced mammary tumors in rats. Breast Cancer Res Treat 1996;39:7-20.

21. World Health Organization. World Health Organization Classification of Tumours. Pathology and genetics of tumours of the breast and female genital organs. Lyon: IARCPress; 2003. p.64-7.

22. Rosen PP. Rosen's breast pathology. $2^{\text {nd }}$ ed. Philadelphia: Lippincott Williams \& Wilkins; 200I.

23. Schnitt SJ. The diagnosis and management of pre-invasive disease. Flat epithelial atypia: classification, pathologic features and clinical significance. Breast Cancer Res 2003;5:263-8.

24. Fraser JL, Raza S, Chorny K; Connolly JL, Schnitt SJ. Columnar alteration with prominent apical snouts and secretions. Am J Surg Pathol 1998;22: | 521-27.
25. Alvarenga M. Tumores da mama. In: Billis A, Vassallo J, editores. Patologia diagnóstica de tumores. Campinas; 2004. p.205-98

26. Shaaban AM, Sloane JP, West CR, Moore FR, Jarvis C, Williams EMI, Foster CR. Histopathologic types of benign breast lesions and the risk of breast cancer. Am J Surg Pathol 2002;26:421-30.

27. Thompson HJ, Singh M. Rat models of premalignant breast disease. J Mammary Gland Biol Neoplasia 2000;5:409-20.

28. Briand R. Hormone dependent mammary tumors in mice and rats as a model for human breast cancer. Anticancer Res 1983;3:273.

29. Soderqvist G, Isaksson E, Schoiultz B, Carlstrom K, Tani E, Skoog L. Prolifaration of breast epitelial cells in healty women during the menstrual cycle. Am J Obstet Gynecol 1997; 176: 123-8.

30. Sedgwick CJ. Allometric scaling and emergency care: the importance of body size. In: Fowler ME. Zoo \& wild animal medicine. Philadelphia: W.B. Saunders Company; 1993.

31. Pachaly JR, Brito HFV. Interspecific allometric scaling. In: Fowler ME, Cubas ZS. Biology, medicine, and surgery of South American wild animals. lowa State University: Press/Ames; 200 I. p.475-80.

32. Russo J, Russo IH. Genotoxicit of steroidal estrogens. Trends Endocrinol Metab 2004;5:21 I-4.

33. Lieher JG. Genotoxicity of the steroidal oestrogens oestrone and oestradiol: possible mechanism of uterine and mammary cancer development. Hum Reprod Update 200 1;7:273-81.

34. Shi YE, Liu YE, Lippman ME, Dickson RB. Progestins and antiprogestins in mammary tumour growth and metastasis. Hum Reprod 1994; 9: 1 62-73 Supplement I.

35. Koukoulis GA. Hormone replacement therapy and breast cancer risk. Ann N Y Acad Sci 2000;900:422-8

36. Simpson HW, Cornélissen G, Katinas G, Halberg F. Meta-analysis of sequential luteal-cycle-associated changes in human breast tissue. Breast Cancer Res Treat 2000;63:171-3.

37. King RJB. A discussion of roles of o estrogen and progestin in human mammary carcinogenesis. J Steroid Biochem Mol Biol 199|;39:8 I I-8.

38. Nazário ACP, Lima GR, Simões MJ, Novo NF. Cell kinetic of the human mammary lobule during the proliferative and secretary plase of the menstrual cycle. Bull Assoc Ande 1995;79:23-7.

39. Bässler R. The morphology of hormone induced structural changes in the female breast. Curr Top Pathol 1970;53: I-89.

40. Mustafa IA, Bland KI. Physiologic effects of steroid hormones and postmenopausal hormone replacement on the female breast cancer risk Ann Surg 1998;228:638-5I .

4I. Chen CL, Weiss NS, Newcomb P, Barlow W, White E. Hormone replacement therapy in relation to breast cancer JAMA 2002;287:734-4I.

42. Russo J, Russo IH. Differentiation and breast cancer. Medicina (B Aires) 1997; 57(Suppl 2):81 -91.

43. Grubbs CJ, Farnell DR, Hill DL, Mc Donoug KC. Chemoprevention of Nnitroso- methylurea-induced mammary cancers by pretreatment with 17 b- estradiol and progesterone. J Natl Cancer Inst | 985;74:927-3 I.

44. Russo J, Russo I. Biological and molecular bases of mammary carcinogenesis. Lab Invest 1987;57: I 12-37.

45. Gompel A, Kandouz M, Siromachkova A. The effect of tibolona on proliferation, differentiation and apoptosis in normal breast cells. Gynecol Endocrinol 1997:(Suppl I):7-9.

46. Bortoletto CCR, Gonçalves WJ, Baracat EC, Rodrigues de Lima G, GiusaChiferi MG. Morphological evaluation of the endometrium of postmenopausal estrogen treatment and taking medroxiprogesterone acetate at 2,3 and 4 month. Acta Obstet Gynecol Scand 1997;76:76.

47. Black LJ, Sato M, Rowley ER, Magee DE, Bekele A, Williams DC, et al. Raloxifene $(\mathrm{LY}$ I3948 I HCl) prevents bone loss and reduces serum cholesterol without causing uterine hypertrophy in ovariectomized rats. J Clin Invest 1994;93:63-9.

Artigo recebido: I //05/2005

Aceito para publicação: 06/03/2006 